\title{
Enfermedad de Takayasu
}

\author{
Dres. Carlos Sajeh A. ${ }^{1}$, José Dgo. Arce V. ${ }^{2}$, Mariana Contreras C. ${ }^{3}$ \\ Elizabeth Yưlow M. ${ }^{4}$, Sres. Andrés Molina C. ${ }^{5}$, Eduardo Nieme B.
}

\section{Takayasu Arteritis. A Clinical Report}

\begin{abstract}
A seven years olf female was admitted to Luis Calvo Mackenna Children's Hospital. Her past history was remarkable in that she had had fever for the preceding months. Her physical, examination disclosed no pulses at her right brachial artery. Blood pressure in her left upper extremity was 200/150 and the erytrosedimentation rate was $124 \mathrm{~mm}$. in the first hour. Aortography showed contour irregularities, and the presence of stenosis and dilations subtantiated a diagnosis of Takayasu disease. After treatment with steroids and antihypertensive drugs improvement was noted.

A review of the current theory of the etjology of this disease as well as the modes of treatment and follow up are included.
\end{abstract}

En 1908, Takayasu, un oftalmólogo japonés, publicó los cambios en el fondo de ojo de una mujer joven consistentes en alteraciones del flujo de los vasos centrales de la retina ${ }^{1}$. Con el tiempo, hallazgos de ausencia de pulso en las extremidades, asociados a los cambios oculares descritos por Takayasu, llevaron a configurar la entidad patologica que lleva su nombre ${ }^{2}$.

En 1952 Shimizu y Sano presentan una descripción detallada de una arteritis braquiocefálica y denominaron la enfermedad como "Síndrome de ausencia de pulsos" 3 . Este término se hizo común en la literatura mundial y ha llegado a ser sinónimo del Sindrome del arco aórtico, una forma de arteritis obstructiva circunscrita a esa zona. En las décadas siguientes se reunió suficiente evidencia en material de autopsia y arteriografías que demuestra que la arteritis compromete también la aorta torácica y abdominal ${ }^{4}$. En el último tiempo investigadores hindúes y japoneses han proporcionado convincente evidencja clínica, arteriográfica e histopatológica que muchas de las coartaciones aórticas atípicas corresponden a localizaciones variables de arteritis de Takayasu ${ }^{3-4-5-6}$.

La enfermedad, que es más frecuente en mujeres jóvenes 8 - 9 , es una arteriopat ia inflamatoria crónica, de origen desconocido, que produce estrechamiento arterial, hecho que explica su sin-

1 Unidad de Nefrología, Hospital Luis Calvo Mackenna. Departamento de Pediatría - Facultad de Medicina - U. de Chile.

2 Departamento de Radiología, Hospital Luis Calvo Mackenna.

3 Becado de Pediatría, Hospital Luis Calyo Mackenna.

4 Sub-Departamento de Inmunología - Ints. de Salud Pública de Chile.

5 Internos de Pediatría - Facultad de Medicina - División Clencias Médicas Oriente - Universidad de Chile. tomatología, caracterizada por déficit de pulso en extremidades, hipertensión arterial, disnea de esfuerzo, soplos vasculares y sintomas inespecíficos como astenia, baja de peso y fiebre. El dolor, sintoma agudo, es reflejo de la isquenia producida por el déficit circulatorio local ${ }^{9}$. Los cambios en el fondo de ojo (anastomosis arteriovenosas retinales alrededor de la papila), han sido vistas en las grandes revisiones entre el 22 y $41 \%$ de los casos 10 .

En la arteria aorta y ramas mayores, principales localizaciones de la enfermedad, se observa formación de aneurisma y trombos secundarios a fenómenos oclusivos. La microscopía revela marcada fibrosis y engrosamiento de la íntima y adventencia, gran destrucción de las fibras elásticas y musculares de la media, siendo estos tejidos reemplazados por fibrosis y abundante infiltración linfocitaria.

El daño observado es producido por endoarteritis obliterativa de los vasa vasorum 11-12.

Nuestro propósito es presentar una paciente de 7 años con las características descritas en la enfermedad de Takayasu y hacer una revisión de la fisiopatología, tratamiento y pronóstico del cuadro.

\section{CASO CLINICO}

D.L.M., escolar de sexo femenino de 7 años, sin antecedentes de importancia, ingresa al Servicio de Pediatria del Hospital Luis Calvo Mackenna en Noviembre de 1980. Su historia comienza 2 meses antes, con anorexia, baja de peso, alza febril $\left(38^{\circ} \mathrm{C}\right)$ intermitente y de predominio nocturno, dolor abdominal vago y cefalea ocasional.

En el examen físico, llama la atención los valores de presión arterial, que promediaban los $200 / 150 \mathrm{~mm}$. $\mathrm{Hg}$. Pulsos periféricos asimétricos, tal como se observa en el cuadro siguiente: 
Pulso:

Derecho

Temporal
Carótida
Braquial
Radial
Femoral
Pedio

Soplo y aneurisma de carótida derecha, soplo paraumbilical izquierdo. Hígado palpable a $1 \mathrm{~cm}$. bajo el reborde costal de consistencia normal, proyección $6 \mathrm{~cm}$. Bazo palpable en reborde costal. proyección $4 \mathrm{~cm}$. Velocidad de sedimentación 124 mm. en la hora.

Dado el examen fisico $y$ velocidad de sedimentación se sospecha enfermedad de Takayasu, y se realizan los siguientes exámenes:

N. ureico, creatinina plasmatica y clearence de creatinina: normales.

V.D.R.L. negativo.

lg. G, IgA e IgM: dentro de límites normales, sólo con elevación de lgM.

Prueba de latex. Ac. Antinucleares, Dinitroclorobenceno, Rosetas E., linfocitos E., Rosetas E A C, linfocitos B: normales.

\section{Antígenos Presentes:}

H L A: A 11 - X1/B5 - B8. No se determinó series $C, D$ ni $D R$ por no disponer en nuestro medio de antígenos.

P.P.D.: $11 \mathrm{~mm}$. Baciloscopía Negativa en ? oportunidades.

E.C.G.: Reacción inicial de Ventricula izquierdo.

Fondo de ojo: normal.

Radiografía de Tórax: Sin alteraciones.

Oscilometría: Normal en brazo izquierdo y piernas. Brazo derecho, oscilación minima (menos de 1/8), no se palpa pulso axilar ni humoral.

Ecoaor tograf ía abdominal: normal.

Pielografía de eliminación, columna y pelvis: normal.

Ambos riñones son de forma y posición normal. El derecho mide 9,7 por $4,2 \mathrm{~cm}$. El izquierdo es más pequeño, mide 9 por $3,5 \mathrm{~cm}$. La eliminación del medio de contraste es simultánea y en buena concentración. Los aparatos pielocalicilares y uretéres son de aspecto normal. Vejiga de paredes lisas y regulares, sin signos de lesión orgánica.

- Aortografia total: Se observó una alteración difusa del tronco braquiocefálico y carótida derecha, que presentan un calibre irregular, con algunas zonas de dilatación saculares a poco de su origen. A nivel del ostium, se ve una zona de relativa estrechez. Hay obstrucción de la subclavia derecha en su origen. No se observó reflujo de medio de contraste a ventrículo izquierdo. (Fig. 1). Existe además una alteración similar a la descrita, en el sector de la carótida izquierda.

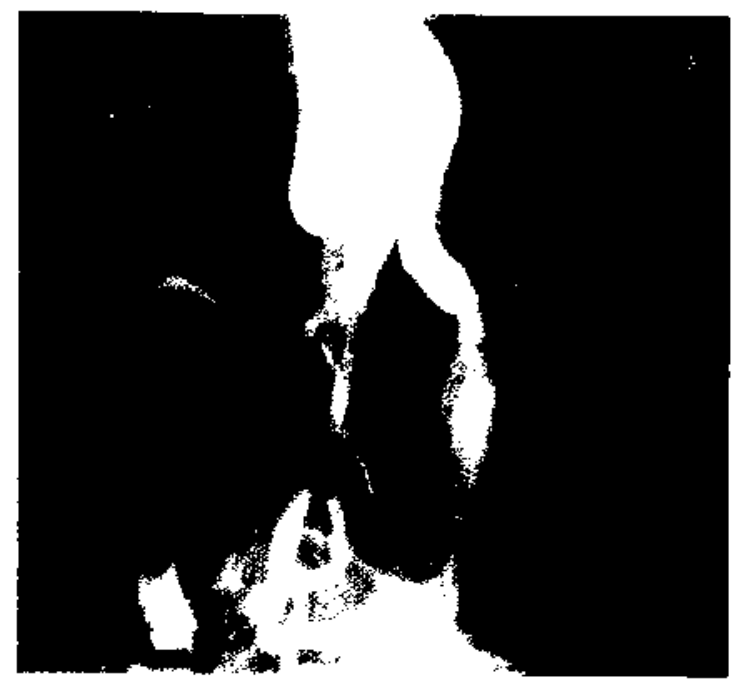

Figura 1.

Aortografía: Arco aórtico

La aorta descendente, muestra una marcada estenosis filiforme. de la arteria renal izquierda, observánduse además, una disminución de tamaño del rišón correspondiente. (Fig. 2).

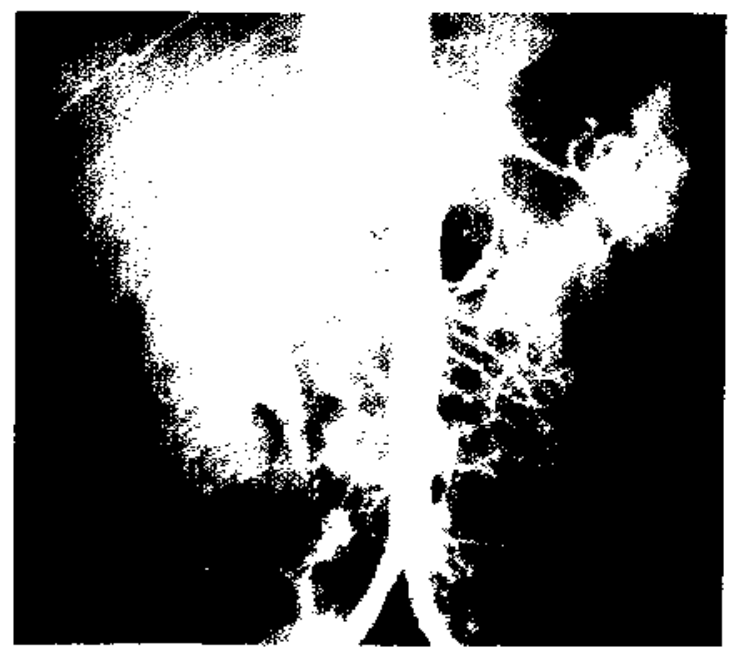

Figura 2.

Aortografia: Aorta descendente

Desde ramas intercostales bajas, se observan colaterales finas, algo tortuosas, que se anastomosan con la axilar derecha por irrigar el brazo de ese lado.

\section{EVOLUCION Y TRATAMIENTO}

Una vez confirmado el diagróstico, se inicia 
tratamiento con prednisona $2 \mathrm{mg} . \mathrm{x} \mathrm{Kg} . \mathrm{x}$ día, por un lapso de 3 meses. A partir de esa fecha, comienza a disminuírse, hasta dejar una dosis de mantención de 7,5 mg. $x$ día. Con este tratamiento, la velocidad de sedimentación, se ha mantenido dentro de límites normales. Desde el punto de vista clínico, no ha tenido molestias, excepto en una oportunidad en que, luego de la suspensión de la prednisona, reaparecen la velocidad de sedimentación elevada y dolor en extremidad superior derecha, por lo que se reinicia tratamiento. (Fig. 3).

Respecto a los pulsos periféricos, desapareció el frémito y aneurisma en cuello. La intensidad de los pulsos ha sido variable, sin desaparecer, excepto en arteria radial y braquial derecha, en donde nunca se han palpado.

En cuanto a la hipertensión, se inició tratamiento con régimen sin sal, hidroclorotiazida y propranoiol, en dosis habituales, consiguiéndose una leve disminución de la presión, pero, presentando oscilaciones, junto con cefalea matinal, por lo que se agrega hidralazina, con to que se consigue mantenerla dentro de límites normales. (Fig. 3).
Por el hecho de mantenerla en tratamiento permanente con corticoides, se le agregó. HIN en forma profiláctica.

\section{COMENTARIO}

El caso que presentamos, una nifia de 7 años, cuya principal sintomatología, hipertensión arterial y déficit de pulsos en una extremidad, se acompañó de velocidad de eritrosedimentación elevada, nos hizo pensar en una enfermdad de Takayasu, de acuerdo a lo señalado en las grandes series $^{4} \cdot 20$. Las más recientes evidencias arteriográficas indican que la aortitis estenosante es únicamente característica de la arteritis de Takayasu y las otras formas están primariamente asociadas con dilataciones de la aorta y formación de aneurismas. Sin embargo en algunos casos puede presentarse la enfermedad de Takayasu sólo como forma aneurismática, lo cual dificulta el diagnóstico, ya que el aspecto arteriográfico puede ser indistinguible de aortitis de otras etiologias ${ }^{13}$. De gran ayuda puede ser el estudio histopatológico en el diagnóstico diferencial de la aortitis, aunque

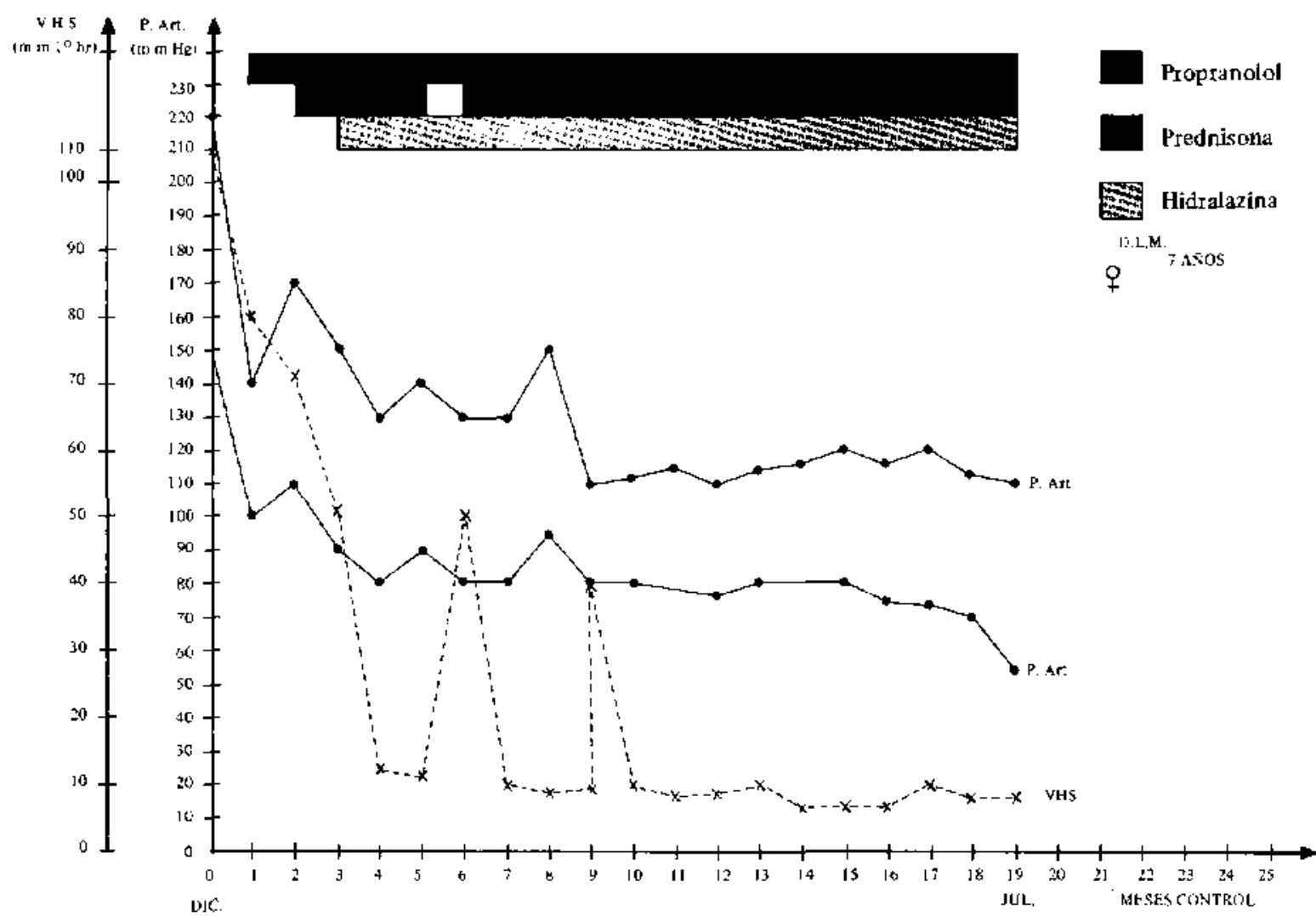

Figura 3.

Tratamiento $y$ Fvolución 
pensamos que la biopsia arterial no debe ser realizada en un paciente portador de una enfermdad cuyas arterias constituyen un tejido inflamado, que hará de este procedimiento un grave riesgo. Creemos que sólo es posible realizar este estudio cuando es preciso realizar una corrección quirúrgica, aprovechando la oportunidad para enviar la muestra al patólogo.

La aortografía, el más importante método diagnóstico, confirmó en nuestra paciente los hallazgos comunicados en otras series: dilataciones prestenóticas e irregularidades pequeñas del lumen en aorta descendente y arco, oclusión de la arteria subclavia, aorta torácica descendente con aneurisma saculares y fusiformes, áreas de contornos irregulares, estrechamiento del lumen distal en el origen de las grandes ramas (imagen descrita como "Cola de ratón" o "Coma"), aorta abdominal con aneurismas preferentemente fusiformes, estrechamiento y marcada irregularidad del lumen que compromete tronco celíaco, arteria mesentérica y arterias renales. En algunos casos se observa circulación supletoria colateral ${ }^{13-14-15}$. En la radiografía de tórax y electrocardiograma se observa crecimiento de ventrículo izquierdo ${ }^{16}$, hecho que en nuestro caso sólo observamos en el último examen mencionado y en grado inicial.

La etiología de la enfermdad permanece aún oscura, aunque se han hecho intentos para relacionarla con ciertas patologias, siendo las principales: Sífilis, tuberculosis, espondilitis anquilosante, sindrome de Reiter, artritis reumatoídea, etc.

La relación con tuberculosis ha sido investigada por muchos autores, varios de los cuales han observado la coexistencia de TBC. pulmonar y extrapulmonar con Arteritis de Takayasu, algunos autores 10 encontraron un $48 \%$ de infección tuberculosa previa, como adenopatia, TBC. pulmonar y eritema indurado, observación confirmada por una revisjón reciente de 7 series. 17 .

Se han detectado desórdenes inmunológicos en esta enfermedad, lo que ha permitido postular un mecanismo autoinmune como factor etiológico, apoyado por recientes hallazgos de laboratorios como aumento de gamaglobulina circulante, antjcuerpos antiaorta circulante y la naturaleza sistémica de la enfermedad ${ }^{10}$. Sin embargo un estudio reciente ${ }^{1 a}$ no demostró que los complejos inmunes circulantes fueran la causa primaria de la enfermedad, pero ellos pueden acelerar o modificar su estado fisiopatológico.

En nuestra paciente se investigaron sífilis, tuberculosis y desórdenes inmunológicos, resultando todos los exámenes normales.

Un factor genético en el origen de la enferme- dad ha sido exhaustivamente investigado por $\mathrm{Nu}$ mano y cols. ${ }^{8}$, quienes encontraron en un estudio de 65 casos una asociación significativa de enfermedad de Takayasu con los antigenos HLA A10, y B5 respecto a la población normal. En el mismo estudio el análisis de 6 familias con más de un caso de enfermedad de Takayasu reveló una tendencia a la asociación de la enfermedad con el fenotipo HLA A9 - A10/B5-B40 apareciendo como más constante el haplotipo A9-B40 en los sujetos portadores de la patología.

Aunque en nuestra paciente se encontró el haplotipo Al1-B5, también comunicado por Numano como asociado a la enfermedad, su presencia sólo confirma los hallazgos del citado estudio realizado en Japón y no puede considerarse como apoyo điagnóstico.

El riesgo relativo de enfermedad de Takayasu en los portadores de B5 es de 3.6. Para considerar la asociación con HLA como una herramienta diagnóstica, el riesgo relativo debe ser alto, como ocurre, por ejemplo, en la asociación del HLA. B27 con la espondilitis anquilosante, en que el riesgo relativo es de 90,1 en población caucásica.

Es interesante señalar, sin embargo, el que nuestra paciente tiene el antígeno BS, yä que el citado estudio sobre HLA fue realizado en población japonesa, genéticamente distinta de la chilena, aunque la frecuencia del BS en la población normal es semejante: $28 \%$ en Chile ${ }^{19} 32 \%$ en $\mathrm{Ja}$ pón 20 . Ello podría apuntar a la existencia de una real asociación de la enfermedad con el HLA-B5, aunque estudios recientes en población caucásica de EE.UU. no demostró asociación con Ags HLA - A y B, 20 .

La asociación de DR4 y DR3 con otras patologías autoinmunes es cada vez más evidente, lo que sugiere que genes muy ligados a ellos y en el caso de Takayasu a MB3, predisponen al paciente a la enfermedad.

E1 B5 podría, por lo tanto ser un marcador directo, por estar en desequilibrio de unión, de alguno de los genes DR. o MB indicadores de susceptibilidad. Lamentablemente, en nuestro país aún no disponemos de los medios para estudio de DR y aloantígenos de células $B$.

Con respecto a tratamiento, son pocas las publicaciones que a él se refjeren $4 \cdot 10 \cdot 12$. Los corticoides, las drogas antituberculosas y la cirugia son las que se mencionan. El empleo de corticoides se ha hecho en forma empírica y con resultados variables. En nuestro caso con el uso de prednisona se observó desaparición del aneurisma carotídeo, mejoria en los pulsos de las extremidades, excepto en brazo derecho donde, sin embargo desapareció el dolor. La velocidad de eritrosedimentación disminuyó en concordancia con el uso de predni- 
sona, al suspenderla temporalmente produjo elevación de la sedimentación y reaparición del dolor, sobre todo en extremidad supetior derecha.

Algunos autores recomiendan el uso de antituberculosos en paises con alta incidencia de TBC., con resultados inciertos 17 .

Como coadyuvantes se han usado antihipertensivos, digitálicos y saliḉlicos. En nuestra paciente el uso de propranolol e hidralazina ha permitido mantener cifras tensionales dentro de límites normales.

B tratamiento quirúggico es el menos recomendado por su alta morbimortalidad, por realizarse en tejidos alterados como es el inflamatorio, por persistencia de la estenosis o reestenosis en la zona de la operación o en otra zona y porque no existen publicaciones de seguimiento a largo plazo.

Su indicación es motivo de análisis en cada caso individual por un equipo multidisciplinario.

La velocidad de eritrosedimentación (VHS) es el examen de laboratorio que nos ha permitido el control del tratamiento de la enfermedad, debido a la buena correlación observada entre el uso de prednisona y la disminución de ella, como asimismo su elevación al suspenderla, y fundamentalmente por su relación con la clínica. Un gran inconveriente de este examen es su irespecificidad, ya que puede elevarse sin que ello signifique actividad de la enfermedad. Como ejemplo, en la Figura 3 podemos ver una significativa elevación. de la V.H.S., que coincidió con una otitis media aguda, volviendo a valores normales luego del tratamiento.

El pronóstico de la enfermedad es incierto. El conocimiento actual de ella sólo permite modificar algunos de sus síntomas, por lo que se recomienda controles periódicos y seguimiento de por vida.

\section{RESUMEN}

Se presenta el caso de una nifía de 7 años con enfermedad de Takayasu confirmada mediante aortografias. Su presión arterial en el brazo izquierdo era de $200 / 150 \mathrm{~mm} \mathrm{Hg}$. al ingreso. Después de tratamiento con corticoides y drogas antihipertensivas pudo observarse cierta mejoría.

\section{ADDENDUM}

En el momento de entregar este manuscrito se realizó el diagnóstico de la enfermedad en una niña de 10 meses de edad.

Paciente de sexo femenino de 6 meses de edad que se hospitaliza por presentar irritabilidad, rechazo de alimentación y fiebre $\left(37.8^{\circ} \mathrm{C}\right)$.

$\mathrm{Al}$ examen se comprueba una presión arterial de 160/120. pulsos amplios y simétricos, con taquicardia incontable.
Se diagnostica insuficiencia cardíaca y comienza a tratarse con Hidroclorotiazida, Propranolol y además se digitaliza. Su evolución es favorable.

La radiografía de tórax, el E.C.C. y la Ecocardiografía demuestran un crecimiento global del corazón.

El fondo de ojo es normal.

P.P.D. y la baciloscopía es (--) VDRL. (-)

Bateria Inmunológica dentro de límites normales.

Antígenos presentes: HLA - Al, HLA - B17 y HLA - B 15 .

No se realizó determinación de las series $\mathrm{C}, \mathrm{D}$ ni DR. por no disponer en nuestro país de los antisueros C ni de los medios necesarios para D/DR.

VHS. $24 \mathrm{~mm}$ en 1 hora.

Aortografía total: Los vasos arteriales del cuello y del tórax son de aspecto radiológico normal.

En aorta abdominal se observa un compromiso difuso desde el tronco celíaco hacia abajo, caracterizado por una disminución del calibre e irregularidades de los bordes. Hay una dilatación de tipo aneurismática a rivel del origen de la mesentérica superior. Estrechez filiforme de la arteria renal derecha y obstrucción completa de la arteria renal izquierda, la cual se llena a través de pequeñas ramas retroperitoneales $y$ de una arteria epigástrica.

El resto de los exámenes ha resultado normal y con diagnóstico de enfermedad de Takayasu se inició tratamiento de su hipertensión, esperando el eventual uso de corticoides en caso de actividad de la enfermedad, la cual la medimos a través de la V.H.S. y la batería inmunológica.

\section{REFERENCIAS}

1 Takayesu M. Case with unusal changes of the central vessels in the retina. Acta Soc. Ophtalmol. Jap. 12, $554 ; 1856$.

${ }^{2}$ Londe A. and Boukman Y. M. Aortitis. Pathology, clinic and arteriographic review. Radiol. Clin. of N.A.

Vol. XIV 2 Pag. 219. 1976.

3 Shimizu $K$. und Sano $K$. Pulseless disease. J. Neuropathol. Clin. 1, 37;1951.

4 Ishikame $K$. Survival and morbidity after diagnosis of occlusive thromboaorthopathy. An. J. Cardiol. 47 (5), $1026 ; 1981$.

S Sen H. P. Obstructive disease of the aorta and its branches. Int. J. Surg. 39, $289 ; 1968$.

6 Nakao L., Inedo M., Kimaio $S$. et al. Takayasu's arteritis: clinical reports on eighty four cases. Circulations 35, 1141;1967.

${ }^{7}$ Donoray J. T. and Ong $H$. E. Primary arteritis of abdominal aorta in children causing bilateral stenosis of renal arteries and hypertension. Circulation 20, $856 ; 1959$.

- Numano F. H L A antigens in Takayasu's disease. Am. Heart J. 98, 153; 1979. 
${ }^{9}$ Ishikawa $K$. Natural history and classification of occlusive thromboarteriopaty. Circulation 57, 27; 1978.

10 Lupi Herrere et ol. Takayasu's arteritis. Clinical study of 107 cases. Am. Heart J. 93, 94; 1977.

11 Rose A.G. Takayasu's arteritis, A. study of 16 autopsy cases. Arch. Pathol. Lab. Med. 104, 231 ; 1980.

12 Wiggelinkhuizen J. Takayasu's arteritis and renovascular hypertension in childhood. Pediatrics 62, 209; 1975.

13 Inoda $K$., Shimizu $H$. et al. Pulseless disease and atypical coartation of the aorta. Arch. Surg. 84, 306; 1962.

14 Mallory B. T. Case record of the Massachussett's General Hospital. New Eng. J. Med. 214, 690; 1938.

15 Loude A., Rossi $P$. The value of total aortography in the diagnosis of Takayasu's arteritis Radiology 114, $287 ; 1975$.

16 Berkmen Y. M. and Laude A. Chest joentgenography as a window to the diagnosis of Takayasu's disease. Radiology 125, 842; 1975.

17 Pantell R.H. and Goodman B. W. Takayasu's arteritis: the relationships with tubcrculosis. Pediatrics 67, 84; 1981 .

18 Numono $F$. Circulating immune complexes in Takayasu's disease. Lack of evidence for a causative role. Arch. Inter. Med. 141, 162, 1961.

19 Yurlow, E. Comunicación personal.

20 Valkman, D. J., Mon D. L. y Fanci A. S, Association between Takayasu's Arteritis and B. cell allo antigens in North Americans. N. England J. Med. 306, 464; 1982. 